\title{
Joule-Level High-Efficiency Energy Transfer to Subpicosecond Laser Pulses by a Plasma-Based Amplifier
}

\author{
J.-R. Marquès, ${ }^{1, *}$ L. Lancia, ${ }^{1, \dagger}$ T. Gangolf, ${ }^{1,2}$ M. Blecher, ${ }^{2}$ S. Bolaños, ${ }^{1}$ J. Fuchs, ${ }^{1}$ O. Willi, ${ }^{2}$ \\ F. Amiranoff, ${ }^{3}$ R. L. Berger, ${ }^{4}$ M. Chiaramello, ${ }^{3}$ S. Weber, ${ }^{5}$ and C. Riconda ${ }^{3}$ \\ ${ }^{1}$ LULI, CNRS, École Polytechnique, CEA, Sorbonne Université, F-91128 Palaiseau, France \\ ${ }^{2} I L P P$, Heinrich-Heine Universität Düsseldorf, 40225 Düsseldorf, Germany \\ ${ }^{3}$ LULI, Sorbonne Université, CNRS, École Polytechnique, CEA, F-75005 Paris, France \\ ${ }^{4}$ Lawrence Livermore National Laboratory, Livermore, California 94550, USA \\ ${ }^{5}$ Institute of Physics of the ASCR, ELI-Beamlines, 18221 Prague, Czech Republic
}

(Received 28 June 2018; revised manuscript received 29 October 2018; published 12 April 2019)

\begin{abstract}
Power amplification and pulse compression of short-pulse laser beams by three-wave nonlinear processes in a plasma was proposed by Malkin, Shvets, and Fisch [Phys. Rev. Lett. 82, 4448 (1999)] as a potential path to exceed the limits of the highly successful technique of chirped pulse amplification [D. Strickland and G. Mourou, Opt. Commun. 56, 219 (1985)] developed three decades ago. The parametric processes considered, Raman and Brillouin scattering, are notoriously difficult to control and to predict theoretically because of the dependence on plasma properties and other nonlinear processes. Previous experiments of backward Raman and Brillouin amplifiers have fallen far short of the predictions of simulations and theory and achieved only a very small energy transfer. In this paper, laser-plasma amplification of subpicosecond pulses above the Joule level is demonstrated with a large energy transfer and a very high efficiency, up to $20 \%$, a major milestone for this scheme to become a solution for the next generation of ultrahigh-intensity lasers. In addition, three-dimensional simulations of the amplification process quantitatively match the experimental results and demonstrate the ability of predictive simulations for the optimization of experiments. The global behavior of the amplification process is reproduced, including the evolution of the spatial profile of the amplified seed, the pulse length of the amplified seed, and the influence of parasitic spontaneous Raman and Brillouin scattering.
\end{abstract}

DOI: 10.1103/PhysRevX.9.021008

Subject Areas: Photonics, Plasma Physics

\section{INTRODUCTION}

The development of high-power, high-intensity lasers in the 1960s led quickly to new ideas in many fields of physics such as inertial confinement fusion, which is now being pursued at the National Ignition Facility. This 2 MJ500 TW facility typically uses pulse lengths of $1 \mathrm{~ns}$ but is capable of making pulses as short as 30 ps. With the development of chirped pulse amplification (CPA) in the 1980s [1] that makes subpicosecond pulses possible, ideas to use laser beams to accelerate particle beams to $\mathrm{GeV}$ or even $\mathrm{TeV}$ energies were pursued [2] that now have attracted the interest of high-energy physics for the next generation

\footnotetext{
*jean-raphael.marques@polytechnique.fr

Also at SBAI, Università di Roma, La Sapienza, via Scarpa 14, 00161 Rome, Italy.

Published by the American Physical Society under the terms of the Creative Commons Attribution 4.0 International license. Further distribution of this work must maintain attribution to the author(s) and the published article's title, journal citation, and DOI.
}

of accelerators. Femtosecond pulses are now accessible to many university-based scientists for research in physics, chemistry, and biology albeit mostly at sub-Joule laser energy. Interest in applications of very intense laser beams (that at some point will very likely exceed the limit of the CPA technology) motivates the current search for new techniques that have the potential to achieve new records in power and energy at a subpicosecond pulse duration. Backward Raman amplification (BRA) in plasmas is such a technique (proposed by Malkin, Shvets, and Fisch [3] and demonstrated by Ping et al. [4]), provided it can overcome several known problems to laser propagation over centimeter scales such as laser filamentation and parasitic spontaneous stimulated Raman (SRS) and Brillouin (SBS) scattering [5].

BRA works by counterpropagating a short "seed" pulse of laser light into a long "pump" pulse of higher-frequency laser light in a plasma whose electron plasma frequency $\omega_{p e}$ equals the difference frequency of the two light waves, $\omega_{0}$ and $\omega_{1}$, respectively. The resonant interaction of these three waves amplifies the seed exponentially until its intensity equals or exceeds the pump one. At that point, 
the leading edge of the pump's power is depleted, so only the front of the seed is amplified. Further interaction depletes the pump and transfers the power to the amplified seed, whose pulse continues to shorten in time.

Backward Brillouin amplification is another technique that works in a similar manner, where the electron plasma wave of frequency $\omega_{p e}=\omega_{0}-\omega_{1}$ is replaced by an ionacoustic wave (IAW) of much lower frequency [6]. The coupling of light with a forced ion mode (strongly coupled SBS [7]) instead of an ion-acoustic wave has a number of advantages: The forced ion response is broadband and allows the amplification of a short seed; moreover, the central frequency of the seed and pump can be the same, which makes experimental realization easier. The energy amplification of a subpicosecond seed beam in this so-called strongly coupled Brillouin regime was originally proposed by Andreev [8] and experimentally demonstrated by Lancia [9]. The optimal parameter range has been assessed [10,11], together with the role of the chirp of the laser [12-14]. The various phases of the amplification process have been quantified $[12,15]$, and the transition to the efficient selfsimilar regime has been experimentally demonstrated [16].

After 18 years of experimental work, the transferred energy and efficiency are far from the theoretical and numerical predictions. Several schemes are proposed to overcome some saturation mechanisms [17-19]. For example, amplification of a $0.3 \mathrm{~mJ}$ seed pulse above $1.2 \mathrm{~J}$ is predicted using the diverging scheme [19], while a $15 \mathrm{~J}-50 \mathrm{fs}$ pulse could be amplified to $5 \mathrm{~kJ}-60 \mathrm{fs}$ from a $10 \mathrm{~kJ}-1 \mathrm{~ns}$ pump in an optimized plasma amplifier [20]. However, with the schemes experimentally explored so far, the maximum energy transfer reported to date is $170 \mathrm{~mJ}$ from a $70 \mathrm{~J}$ pump laser via the Raman scheme [21], corresponding to an efficiency of $0.24 \%$. Using the Brillouin amplification scheme, $60 \mathrm{~mJ}$ have been transferred from a $2 \mathrm{~J}$ pump laser [9], corresponding to a $3 \%$ total efficiency.

In this paper, we demonstrate laser-plasma amplification of subpicosecond pulses above the Joule level, reaching an unprecedented energy transfer of up to $2 \mathrm{~J}$, with a very high efficiency, up to $20 \%$, satisfying for the first time in this field the expectations of the theory and simulations. The three-dimensional simulations use the actual measured spatial and temporal profiles of the pump and seed beams, the plasma profiles from hydrodynamic modeling of the plasma evolution, and the actual angles of propagation between the pump and seed beams. Quantitative agreement is obtained on the transferred energy as well as predictions of the deleterious effects of spontaneous SBS and SRS, also observed experimentally. The global behavior of the amplification process is well reproduced, including the evolution of the spatial profile of the seed. We show that the main sources of loss of pump energy available for transfer, in particular, spontaneous Raman and Brillouin backscattering, can be controlled by an appropriate choice of the pump and seed timing as well as initial seed intensity.
We also present the first investigation of Brillouin laser amplification as a function of the incident seed intensity. Exploring 6 orders of magnitude, up to intensities exceeding the pump, we reveal the importance of using a high incident intensity to allow access to the self-similar regime as fast as possible and obtain a highly efficient energy transfer in a subpicosecond scale. In this regime of efficient amplification, we also observe evidence of energy losses of the seed by spontaneous backward Raman.

\section{EXPERIMENTAL SETUP AND METHOD}

The results presented here have been obtained at LULI on the ELFIE facility. A schematic view of the experimental setup is shown in Fig. 1. A "pump" and a "seed" beam, of the same wavelength $\left(\lambda_{0}=1057 \mathrm{~nm}\right)$, linearly polarized, cross in a plasma at an angle of $165^{\circ}$ in the $y z$ plane. The seed is focused at its minimum focal spot $\sigma_{x} \times \sigma_{y} \simeq 100 \times 60 \mu \mathrm{m}^{2}$ [full width at half maximum (FWHM)] and compressed to its minimum duration (FWHM $\sim 0.55 \mathrm{ps}$ ). Its maximum energy is approximately $4 \mathrm{~J}$, providing a maximum intensity of $I_{0}^{\max } \sim 10^{17} \mathrm{~W} / \mathrm{cm}^{2}$. To increase the interaction volume, the $9 \mathrm{~J}$ pump is defocused and stretched, to a focal spot of $\sigma_{x} \times \sigma_{y} \simeq 85 \times 135 \mu \mathrm{m}^{2}$ (FWHM) and a duration of 1.7 ps FWHM [red before blue, $\omega(t)=\omega_{0}\left[1-2 \alpha \omega_{0} t\right]$, $\alpha=-7.65 \times 10^{-7}$ ], leading to a maximum intensity of $I_{1}^{\max } \sim 4 \times 10^{16} \mathrm{~W} / \mathrm{cm}^{2}$. The plasma is created $1 \mathrm{~ns}$ before pump and seed arrival by a third beam (35 J-500 ps$1057 \mathrm{~nm})$ propagating at $135^{\circ}$ from the pump. This beam is focused to a $\sigma_{x} \times \sigma_{z} \simeq 200 \times 1200 \mu \mathrm{m}^{2}$ spot, reaching an average intensity of approximately $2 \times 10^{13} \mathrm{~W} / \mathrm{cm}^{2}$ that fully ionizes a hydrogen supersonic gas jet. The resulting plasma density profile is Gaussian $\left(\sigma_{y} \times \sigma_{z} \simeq 750 \times 575 \mu \mathrm{m}^{2} \quad \mathrm{FWHM}\right)$ with a maximum electron density adjusted to $n_{e} \simeq 4-5 \times 10^{19} \mathrm{~cm}^{-3}$ $\left(n_{e} / n_{c} \sim 0.04-0.05\right.$, where $n_{c}$ is the critical density at the laser wavelength). The electron and ion temperatures at pump and seed arrival are $T_{e} \sim 100 \mathrm{eV}$ and $T_{i} \simeq 80 \mathrm{eV}$ (from 2D fluid simulations with the code FCI2 [22]). Let us note that in the strong coupling regime, even if $T_{e} \approx T_{i}$, due to their large phase velocity, Landau damping on ion perturbations will be relatively small. At the plasma density and temperature of the present experiment, the pump intensity threshold for the strongly coupled regime is $I_{0}^{\text {th }} \sim 10^{13} \mathrm{~W} / \mathrm{cm}^{2}$, reached very early in the pump leading edge. The pump and seed waves are at the same frequency with a relatively narrow bandwidth $\left(\Delta \omega / \omega_{0} \sim 5 \times 10^{-3}\right)$. The time delay $t_{0}-t_{1}$, where $t_{0}$ and $t_{1}$ are the arrival times at the plasma center of the maximum of the pump and seed beams, respectively, is adjusted with a delay line (for $t_{0}-t_{1}>0$, the seed arrives before the pump).

Let us note that a fully counterpropagating $\left(180^{\circ}\right)$ geometry would have been better for an optimum coupling between the pump and seed, as demonstrated in Ref. [16]. However, the incident seed intensity in Ref. [16] is $I_{1}^{\text {inc }} \sim 10^{13} \mathrm{~W} / \mathrm{cm}^{2}$ 


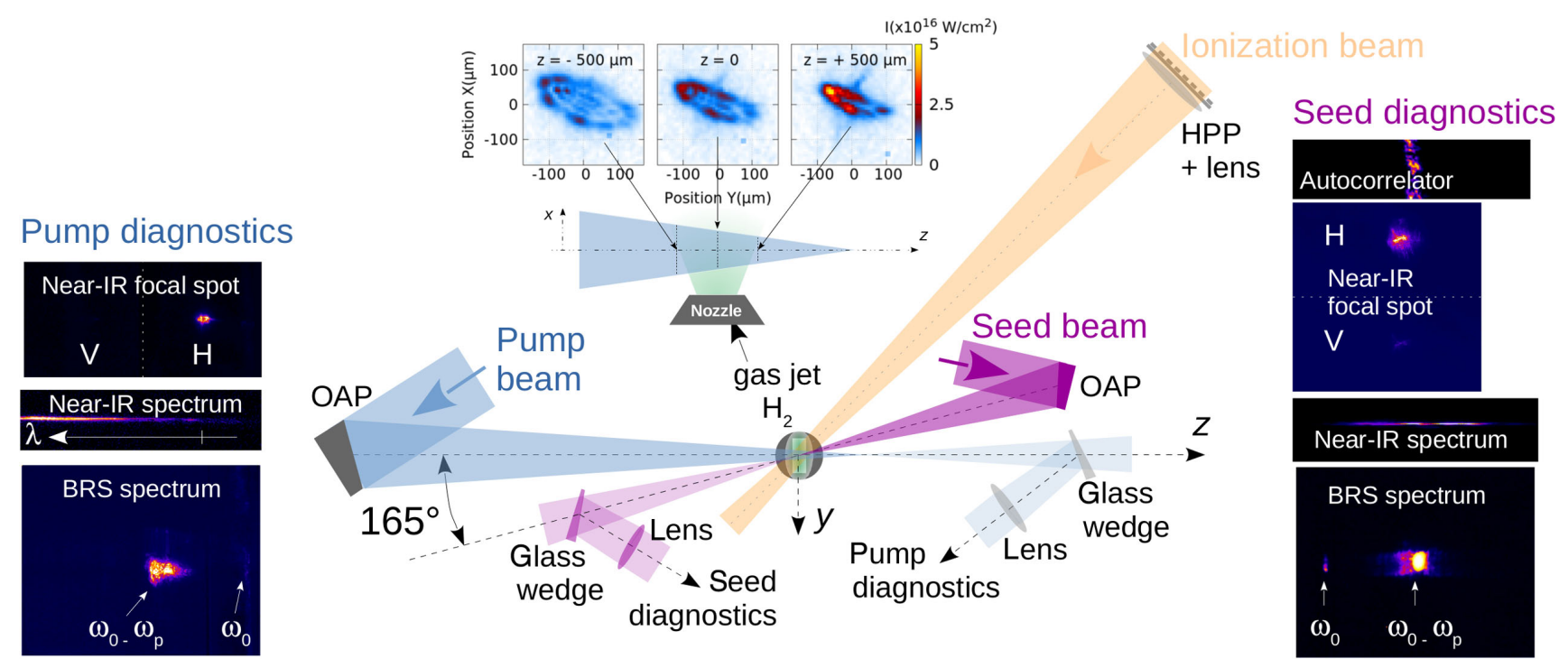

FIG. 1. Experimental arrangement, showing pump and seed beams focused by off-axis parabola (OAP), the ionization beam focused using a lens after a hybrid phase plate (HPP), the supersonic hydrogen gas jet at the beam intersection, the glass wedges sending a lowintensity fraction of the beams to lenses imaging the beam focii toward their diagnostics, raw images of each diagnostics, and transverse profiles of the pump at three positions in the gas jet $(z=-500,0$, and $+500 \mu \mathrm{m}$ from the plasma center). Pump and seed diagnostics are described in Sec. II.

(a few $\mathrm{mJ})$. In the present experiment, to explore interaction at much higher incident seed intensities $\left(I_{1}^{\text {inc }}\right.$ up to $10^{17} \mathrm{~W} / \mathrm{cm}^{2}$, a few Joules) without risking damage to the laser chain with counterpropagating beams, we have to use a $165^{\circ}$ geometry, even if it reduces the interaction length, the quality of the transverse overlap, and the control of pump spontaneous Raman scattering.

The focal spots and spectra of the transmitted pump and seed beams near their central wavelength (1050-1070 nm) are recorded on high-dynamic CCD cameras. The infrared backward $\left(165^{\circ}\right)$ Raman spectra $(1100-1700 \mathrm{~nm})$ of each beam are recorded on InGaAs CCD cameras, providing the amount of Raman signal and allowing the retrieval of the electron plasma density. In order to measure the transmitted pump and seed energies, the focal spot diagnostics are absolutely calibrated. The pulse duration of the transmitted seed is measured with a second-order autocorrelator coupled to a high-dynamic CCD camera.

The growth rate of strongly coupled Brillouin (responsible for laser amplification), as well as those of spontaneous Raman or filamentation (responsible for beam losses or deterioration), depends on the plasma density. All the following results are at $n_{e} \sim 0.05 n_{c}$, where we measure the maximum energy transfer. Also, they all correspond to pump and seed pulses synchronized at the center of the plasma.

\section{EXPERIMENTAL RESULTS}

\section{A. Amplification as a function of seed incident energy}

The influence of the incident seed intensity on the final energy transfer (energy gain) is studied by changing only the input seed energy $\left(5 \times 10^{-6}\right.$ to $4 \mathrm{~J}$, half of the pump energy), all the other pump, seed, and plasma parameters being fixed. Figure 2 presents the seed focal spot at the output of the plasma, after interaction with the pump, for different incident seed energies. For every image in Fig. 2, the labels indicate the seed incident and output energies. These values are reported in Fig. 3, together with "test" shots: without the pump or with orthogonal pump and seed polarizations. The filled area in Fig. 3(a) represents the separation between gain (outside the area) and loss (inside). From these two figures, one can see that, when the incident seed energy is increased from $5 \times 10^{-6}$ to $0.2 \mathrm{~J}$ ( $I_{1}^{\text {inc }}$ from $1.6 \times 10^{11}$ to $6.4 \times 10^{15} \mathrm{~W} / \mathrm{cm}^{2}$ ), the amount of energy transferred from the pump to the seed increases up to a value of $\Delta E \sim 1.75 \mathrm{~J}$, which is $20 \%-25 \%$ of the total pump energy. Such an efficiency indicates that the interaction occurs in the pump depletion regime, as expected from selfsimilar Brillouin amplification. To our knowledge, this is an unprecedented level of energy transfer and efficiency from a laser-plasma amplifier of subpicosecond pulses.

As expected from the three-wave coupling, when pump and seed propagate in a plasma with orthogonal polarizations (green square), they cannot couple, and the transferred energy drops. A small transfer is still observed due to the nonperfectly defined polarization of the beams.

When the seed incident intensity is larger than the pump one ( $I_{1}^{\text {inc }} \geq$ few $10^{16} \mathrm{~W} / \mathrm{cm}^{2}$, corresponding to an incident seed energy $E_{1}^{\text {inc }} \geq 1 \mathrm{~J}$ ), the efficiency of the amplification process drops. The seed transmission falls to $50 \%$. It is interesting to compare this situation with a case where no coupling occurs (orange triangle, $t_{0}-t_{1}=11 \mathrm{ps}$, pump 

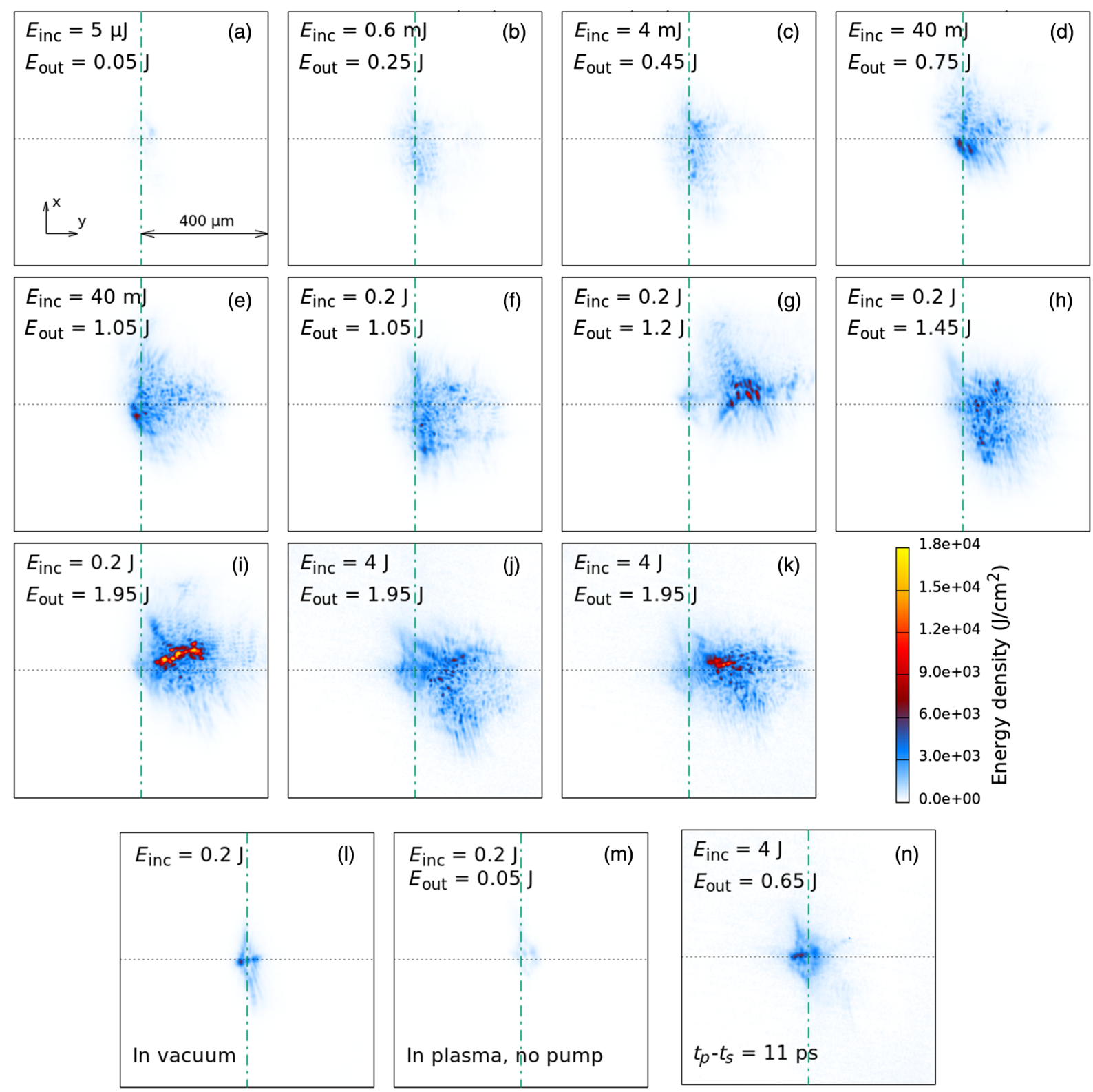

FIG. 2. Transverse profile of the transmitted seed pulse (energy per unit area of the focal spot) (a)-(k) for different incident seed energies, (l) in a vacuum, (m) in a plasma without a pump, and (n) in a plasma with the pump arriving 11 ps after the seed $\left(t_{0}-t_{1}=+11 \mathrm{ps}\right)$. Except for (n), the pump and seed reach the center of the plasma at the same time $\left(t_{0}-t_{1}=0\right)$. The spatial and color scales are the same for all the images, as well as the pump and plasma parameters.

late after the seed): Here, transmission drops at $80 \%$. This result indicates that, even in this particular case of unfavorable initial coupling conditions, energy is still transferred from the pump to the seed.

In the self-similar regime, the seed duration and intensity are correlated; the larger the intensity, the smaller the duration [10,23]. Since the input seed duration in our experiment is fixed (550 fs), depending on its incident intensity, the interaction does not start immediately in the self-similar regime, which reduces the efficiency and slows down the compression process. This nonoptimal seed pulse is particularly deleterious near $10^{17} \mathrm{~W} / \mathrm{cm}^{2}$, where its initial duration is much too long for an optimum energy transfer and compression.

The interest of the crossed polarization and the $t_{0}-t_{1}=$ 11 ps cases compared to shots without a pump is that in these configurations the EM coupling is ineffective, while the other phenomena affecting the propagation of the pump and seed pulses are preserved (hydrodynamics, heating, spontaneous instabilities, etc.), allowing us to clearly separate them.

The fluctuations observed in the transferred energy are due to the shot-to-shot variations of laser-plasma parameters, mainly the seed and pump beam pointing stability. These fluctuations are enhanced by the fact that, due to the 

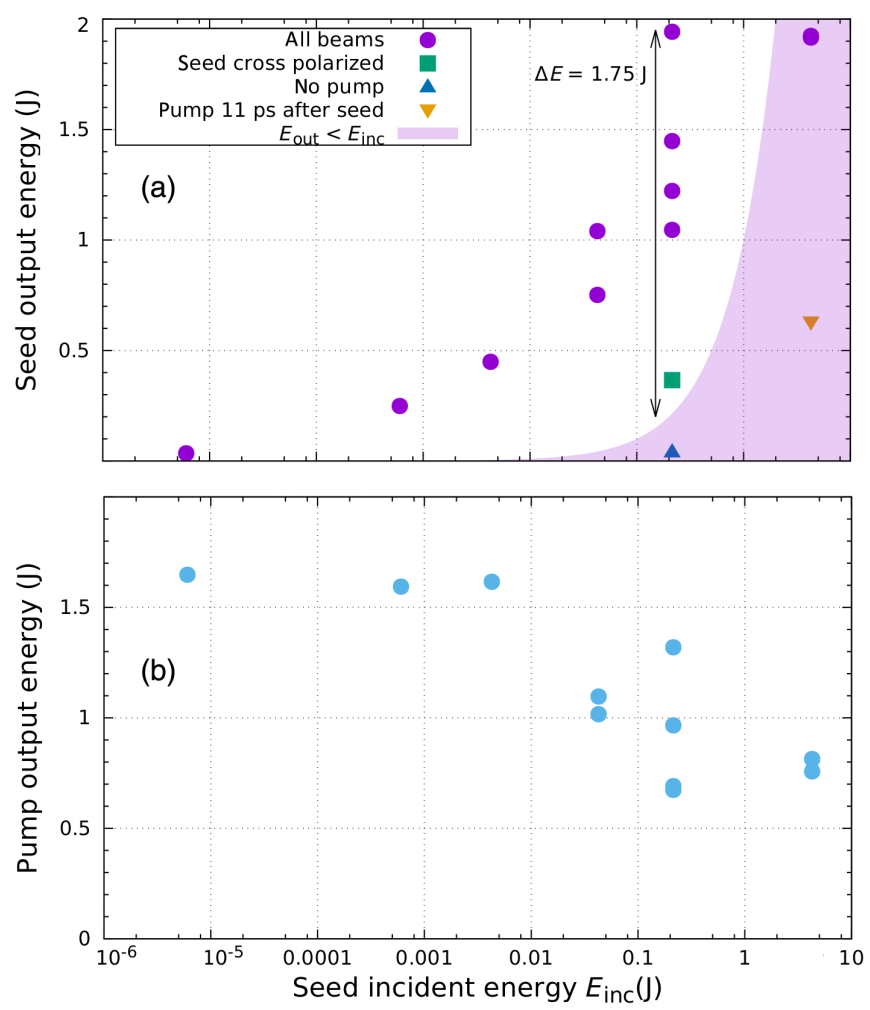

FIG. 3. Energy of the transmitted beams as a function of the incident seed energy $E_{1}^{\text {inc }}$, measured by the integration of the energy-calibrated focal spot images of the seed (a) and the pump (b). The corresponding incident seed intensity in a vacuum is $I_{\text {inc }}\left(\mathrm{W} / \mathrm{cm}^{2}\right) \sim 3.2 \times 10^{16} E_{1}^{\text {inc }}(\mathrm{J})$. Points out of the purple area in (a) correspond to seed amplification. At $E_{1}^{\text {inc }}=0.2 \mathrm{~J}$, the energy gain is $\Delta E=1.75 \mathrm{~J}$. The green square and the orange triangle in (a) are two cases where there is no EM coupling but other mechanisms are present (spontaneous scattering, hydrodynamics, etc.).

experimental arrangement, when the pump moves toward one direction, the seed moves toward the opposite, strongly affecting the overlap.

The transmitted pump energy as a function of the incident seed energy is presented in Fig. 3(b). One can see that the higher the seed energy gain [Fig. 3(a)], the lower the transmitted pump energy. At a very weak incident seed (no energy transfer), the pump-transmitted energy is still quite low, of the order of $1.7 \mathrm{~J}$, which is about $20 \%$ of the incident energy. This relatively low transmission is due to beam refraction and to the losses by collisional absorption and spontaneous Raman instability, both favored by the low plasma temperature $(100 \mathrm{eV})$. On the contrary, when the seed is injected with a high-enough intensity, Brillouin is stimulated and competes with spontaneous Raman instability [16]. Let us note that, because of the losses undergone by the pump before crossing the seed, the available pump energy is between 9 (vacuum) and $1.7 \mathrm{~J}$ (plasma exit), indicating that the amount of effective energy transfer at the interaction point is even higher than $25 \%$.
The other diagnostics (pump and seed spectra, seed autocorrelator) are not calibrated in energy but give the same relative increase of the transferred energy with the injected seed energy, as well as the same relative pump depletion.

The seed spectra are represented in Fig. 4 at different energies of the injected seed. It is shown not only that the amplification happens over the entire seed spectrum, but also that the higher the energy transfer, the broader and more redshifted is the amplified spectrum, a signature of SBS occurring in the self-similar regime [16].

The two uppermost spectra in Fig. 4 correspond to the case where the seed incident intensity is larger than the pump one and the energy transfer is not efficient. However, one can see that, when the seed does not interact with the pump (yellow curve, pump 11 ps after the seed), its spectrum remains centered at its initial vacuum position $(1057 \mathrm{~nm})$ and is not broadened. Moreover, a depletion is observed at the center of the spectrum, which is where the intensity is highest and the SRS and SBS spontaneous instabilities grow fastest. In opposition, when the pump overlaps and couples to the seed, it transfers some energy that compensates part of its losses [orange triangle versus violet circle in Fig. 3(a) at $E_{1}^{\text {inc }}=4 \mathrm{~J}$ ], refilling the seed spectrum (red curve in Fig. 4). Even if the losses are still larger than the energy gain, the interaction occurs

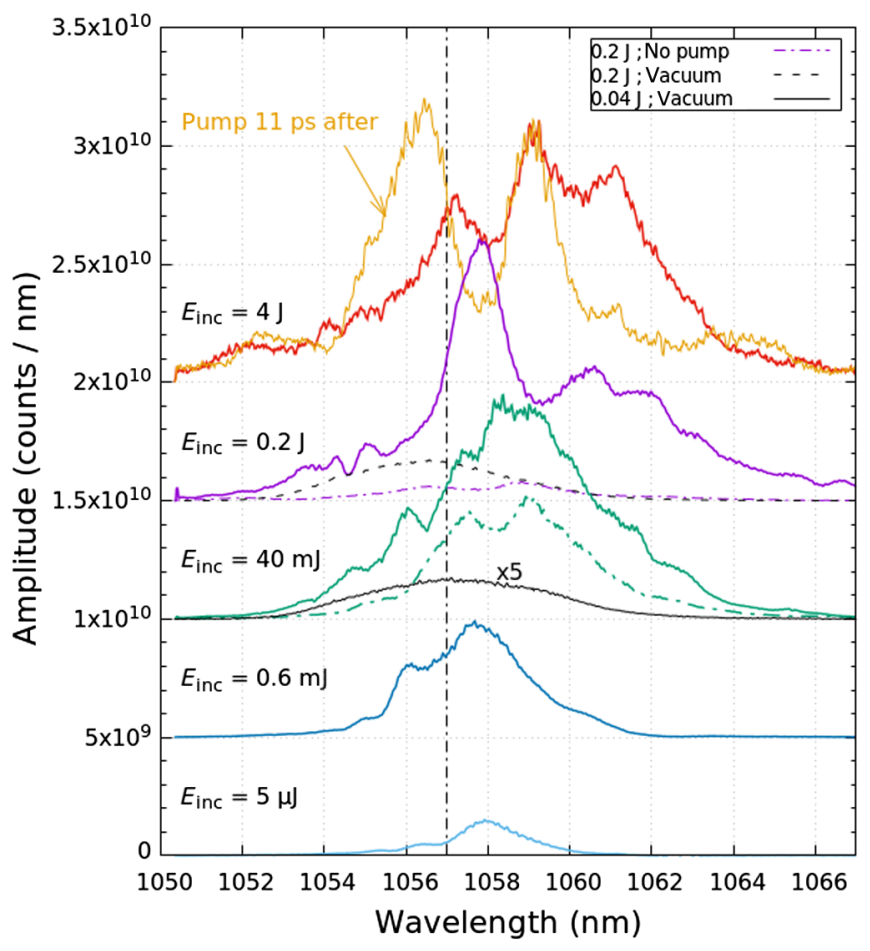

FIG. 4. Transmitted seed spectrum for different incident seed energies. Black lines are reference shots in a vacuum. The spectrum for $E_{1}^{\text {inc }}=40 \mathrm{~mJ}$ in a vacuum (black plain line) is multiplied by a factor of 5 for better visibility. For the same purpose, an offset of $5 \times 10^{9}$ is added to each set of curves. 

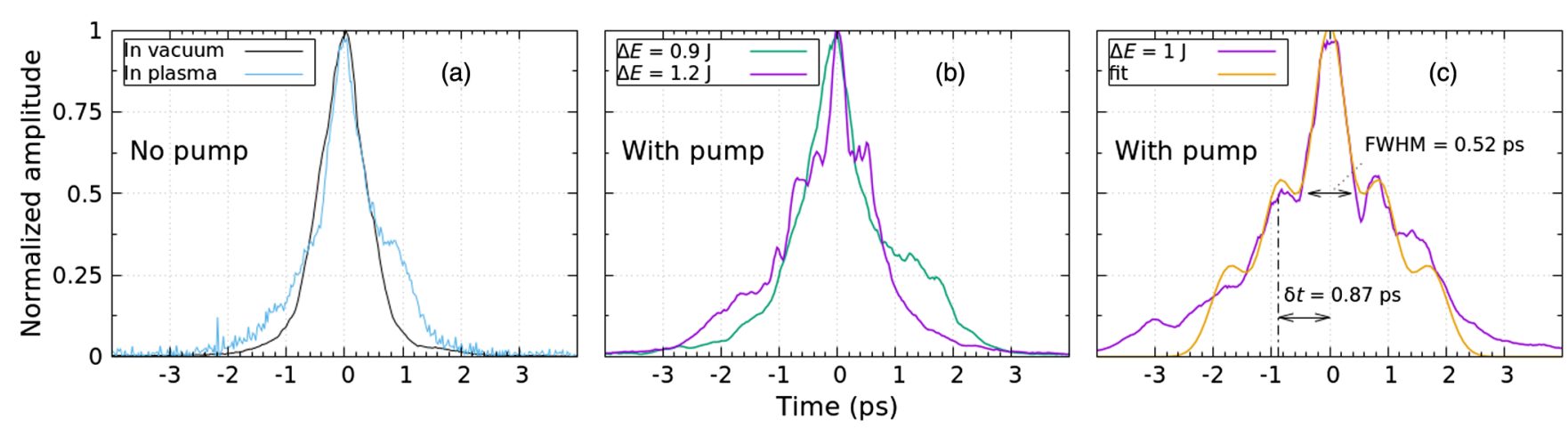

FIG. 5. Autocorrelation traces of the transmitted seed pulse, normalized to their maximum to help the comparison of their width. (a) Without the pump, under vacuum, and in a plasma. (b) In a plasma with a pump, for different transferred energies. (c) In a plasma with a pump, the case with " $\pi$ pulses." The orange curve is a fit using a triple Gaussian function: $e^{-(t / \tau)^{2}}+A\left[e^{-(t+\delta t)^{2} / \tau^{2}}+\right.$ $\left.e^{-(t-\delta t)^{2} / \tau^{2}}\right]+A^{2}\left[e^{-(t+2 \delta t)^{2} / \tau^{2}}+e^{-(t-2 \delta t)^{2} / \tau^{2}}\right] ; \tau=0.43 \mathrm{ps}, \delta t=0.87 \mathrm{ps}$, and $A=0.52$.

in the self-similar regime, redshifting and broadening its spectrum.

\section{B. Transverse profile of the amplified seed}

The bottom images in Fig. 2 show the transmitted seed focal spot in a vacuum (1), and at the plasma exit without (m) and with (i) the pump, at the same incident seed energy $\left(E_{1}^{\text {inc }}=0.2 \mathrm{~J}\right)$. Without the pump, the beam undergoes an attenuation of a factor close to 5, but its transverse profile is not much different from the vacuum case. Conversely, with the pump and at high energy transfer ( $\Delta E \sim 1.7 \mathrm{~J}$, approximately $20 \%$ of the pump energy), the amplification process strongly modifies the focal spot shape and increases its size. The evolution of the seed focal spot is due to (i) the evolution of the pump profile along the interaction region, as shown in Fig. 1 at three positions $(-500,0$, and $+500 \mu \mathrm{m}$ ) along the propagation axis; (ii) the $165^{\circ}$ coupling geometry leading to a progressive transversal drift of the seed pulse through the pump focal spot; and (iii) the amplification process that is nonlinear with the incident seed intensity (Fig. 3). The resulting increase of the focal spot size thus reflects the very high efficiency of the amplification process that makes maximum use of the available pump energy.

On the amplified focal spot, one can observe very small and randomly distributed structures. At our typical pump intensity of $I_{0} \sim 10^{16} \mathrm{~W} / \mathrm{cm}^{2}$, the filamentation growth rate in a hydrogen plasma at $0.05 n_{c}$ is approximately $2 \mathrm{ps}$, about 20 times slower than the sc-SBS growth rate. Filamentation will become significant after a few growth rates, so a few times more than the duration of our pump pulse (1.7 ps) or the time for the pulses to cross the plasma length (approximately $2 \mathrm{ps}$ ). Since the plasma profile is Gaussian, it takes even more time on the lower-density sides of the profile, which is confirmed by $2 \mathrm{D}$ particle-incell (PIC) simulations [24,25] and by 3D-fluid simulations (see below). Moreover, our experimental results confirm these predictions. Indeed, the transmitted focal spot of the pump does not present the small-size, randomly distributed, and shot-to-shot changing pattern characteristic of a beam undergoing filamentation, which is also true for the seed beam when propagating in the plasma without the pump [Figs. 2(m) and 2(n)]. In addition, shots with a pump duration or a plasma length doubled do not show any filamentation of the beam. The very small structures observed in the transmitted seed focal spot appear only when it is coupled to the pump and amplified. We attribute them to the initial spatiotemporal nonuniformities in the amplitude and phase of the pump, seed, and plasma that couple during their propagation and generate this specklelike pattern.

\section{Duration of the amplified pulse}

Autocorrelation traces of the transmitted seed pulse (normalized to their maximum) are presented in Fig. 5. They correspond to the integration along the $x$ axis (the beam vertical extension) of the autocorrelation images (topright image in Fig. 1). As a reference, Fig. 5(a) presents the case of the output seed after propagation in a vacuum (black curve) or in the preformed plasma (blue curve), both without the pump. Figures 5(b) and 5(c) present autocorrelation traces of seed pulses amplified at the Joule level $\left(E_{1}^{\mathrm{inc}}=40 \mathrm{~mJ}\right)$. One can see that the energy of the pump is transferred within the seed pulse envelope (subpicosecond). However, on some shots, the autocorrelation trace presents periodic temporal "satellites" [Fig. 5(c)], indicating the presence of a main pulse followed by others of lower intensities which could be a signature of a main pulse followed by $\pi$ pulses, as expected from amplification in the self-similar regime [12]. Let us note that in this regime the energy transfer is often associated with pulse temporal compression. However, two-dimensional simulations $[23,26,27]$ show that this compression and the position of the pulse maximum are not uniform transversely, leading to an amplified pulse with a "horseshoe" spatiotemporal intensity profile in the focal region (see 3D simulations 
presented in the following). In addition, the traces presented in Fig. 5 are the result of a spatial integration along the transverse (vertical) dimension of the collimated output beam, collected as shown in Fig. 1. It is thus impossible to resolve the on-axis pulse duration. Our autocorrelation results are intended as an upper limit of the output seed duration, and the associated output seed peak intensity is likely underestimated, which is why, for the sake of accuracy, we choose to present the focal spots in Fig. 2 in terms of energy density.

\section{Raman scattering of the amplified seed}

Another signature of the increased seed intensity is found in the enhancement of its Raman backscattered signal. Figure 6 presents, for different incident seed energies, the spectra (a) and energy (b) of the seed Backward Raman scattering (BRS) emitted at $165^{\circ}$. At low incident seed energy, the signal is very weak, indicating that the signal is not coming from forward Raman of the pump. Without the pump, the seed BRS is much weaker [dashed purple curve in
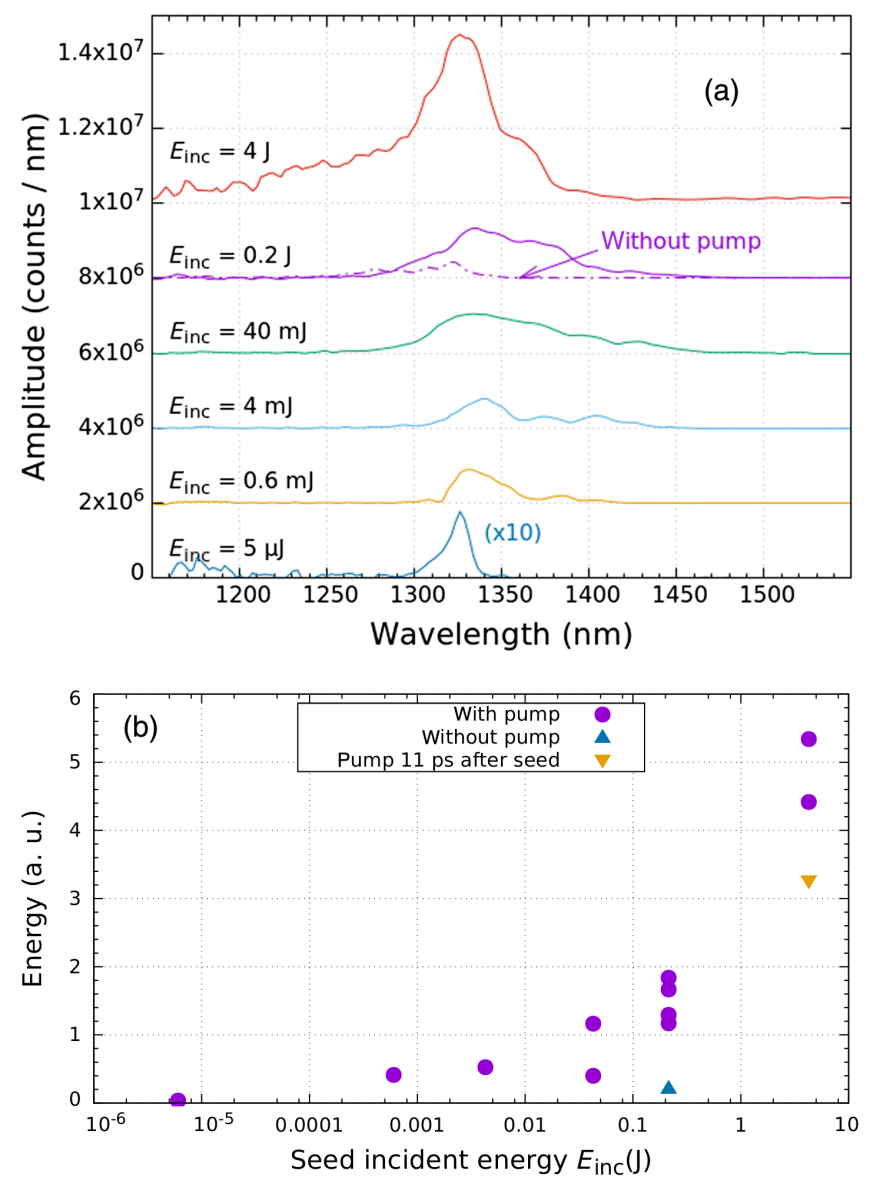

FIG. 6. Seed backward Raman. Spectrum (a) and energy (b) of the seed backward $\left(165^{\circ}\right)$ Raman at different incident seed energies $E_{1}^{\text {inc }}$. In (a), for better visibility, an offset of $2 \times 10^{6}$ is added to each spectrum, and the spectrum at $E_{1}^{\mathrm{inc}}=5 \mu \mathrm{J}$ is multiplied by 10 .
Fig. 6(a) and blue triangle in Fig. 6(b) for $E_{1}^{\mathrm{inc}}=0.2 \mathrm{~J}$, indicating that most of the BRS comes from the intensity increase of the seed. In Fig. 6(a), one can see that the Raman spectra are centered around $1330 \mathrm{~nm}$ and that they never extend closer than $1150 \mathrm{~nm}$ toward the pump and seed wavelength side ( $1057 \mathrm{~nm}$ with a FWHM $=5-6 \mathrm{~nm}$ ), even when $I_{1} \sim I_{0}$, which indicates that amplification of the seed by Raman backscattering of the pump is unlikely.

\section{E. Amplification as a function of pump-seed relative delay}

The maximum energy transfer depends not only on the incident seed intensity, but also on the pump-seed relative delay $t_{0}-t_{1}$, as can be observed in Fig. 7. The maximum energy transfer $(\Delta E \sim 1.2 \mathrm{~J})$ is obtained when the seed reaches the center of the plasma slightly before the pump $\left(t_{0}-t_{1} \sim+1 \mathrm{ps}\right)$. Several scans in pump-seed delay at different pump durations and chirp signs (not the purpose of this paper) show this same behavior. We explain it by the combination of two effects: (i) The amount of spontaneous SRS and SBS undergone by the pump before its interaction with the seed is reduced, preserving the energy available for transfer [10]; (ii) when the seed arrives earlier, it starts to couple to the pump near the maximum of the Gaussian density profile. The low-intensity front part of the pump is thus compensated by the high density of the plasma $\left[\gamma_{s c} \propto\left(I_{0} n_{e}\right)^{1 / 3}\right]$, allowing the self-similar regime to start faster and the coupling to be more efficient [12].

\section{DISCUSSIONS}

In the following, we discuss, with the support of simulations, the different experimental results presented above.

\section{A. Seed amplification in a three-dimensional geometry}

In order to compare the spatial profiles of the amplified seed beam and the total transferred energy, and in order to

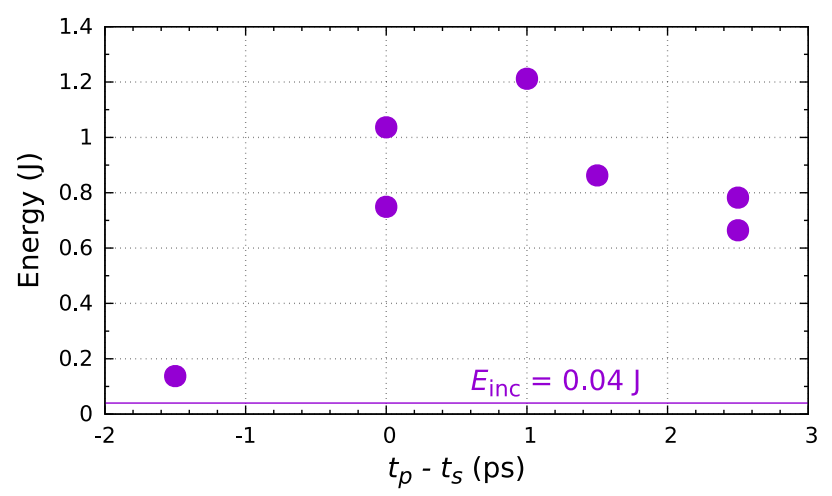

FIG. 7. Seed energy versus pump-seed relative delay. Transmitted energy of the seed as a function of its relative delay with the pump. For $t_{0}-t_{1}>0$, the seed arrives at the plasma center before the pump. 
study the impact of competing instabilities on the amplification process, we ran simulations using the threedimensional wave propagation code $\mathrm{PF} 3 \mathrm{D}[28,29]$. This code treats each light wave in the paraxial approximation. There are separate wave propagation equations for the pump laser light and the Brillouin and Raman amplified light. The Brillouin and pump light are temporally enveloped at the same frequency. The Raman light frequency is chosen by the user to match the conditions of the application. In addition, the light waves are spatially enveloped along the direction of propagation. The Langmuir and ion-acoustic waves are also spatially enveloped. However, the IAW is not enveloped in time, which allows proper treatment of strongly coupled SBS. At the maximum plasma density of $0.05 n_{c}$ and the maximum pump intensity of $I_{0 \text { max }} \sim 4 \times 10^{16} \mathrm{~W} / \mathrm{cm}^{2}$ of this experiment, the sc-SBS growth rate is $\gamma_{s c} / \omega_{0} \sim 4 \times 10^{-3}$ $\left(\gamma_{s c}^{-1} \sim 90 \mathrm{fs}\right)$, justifying the envelope approximation. Let us note that at this relatively high plasma density, and since both pulses have the same frequency, Raman cannot be considered as a coupling mechanism for amplification [24]. However, spontaneous Raman (and Brillouin) scattering of the pump could reduce its energy before it encounters the seed. The spontaneous Raman and Brillouin scattering of the pump and seed beams are included in the simulations, as well as collisional damping and ponderomotive filamentation. Moreover, the effect of the geometry for the beams crossing at an angle (i.e., the effective amplification length in the overlapping region) and the transverse size of the laser beams are fully described. To account for the strong variation of the wave amplitude in the self-similar stage and the $15^{\circ}$ angle of the seed to this axis, the spatial resolutions are $\Delta x=\Delta z=\lambda_{0}$ and $\Delta y=2 \lambda_{0} / 3$. Tests are made to make sure the resolution is adequate. In addition, the axis of propagation is tilted by $7.5^{\circ}$ so both the pump and seed propagate at $\pm 7.5^{\circ}$ to the axis with the same results. The light waves interact with the background plasma through ponderomotive forces which can intensify the light and cause further development of small spatial scales. Because of the short time duration, low plasma density, and low charge state $(Z=1)$, filamentation is not found to be important, and it will not be further discussed. To take into account nonlinear effects on the ion perturbation, such as wave breaking, and in accordance with typical PIC simulation results (see the following), its amplitude is limited to $50 \%$ of the local plasma density. This limit does not affect the amplification of the main part of the seed pulse, for which the density perturbation stays below this limit, but prevents a nonphysical scattering of the pump pulse on these perturbations long after the seed pulse has interacted with the pump [8].

The input laser and plasma parameters are those given in the experimental setup paragraph. The spatial and temporal profiles are Gaussian. The hydrogen plasma density has a peak value of $n_{e} / n_{c}=0.05$, with a FWHM $=575(750) \mu \mathrm{m}$ along the $z(y)$ axis, respectively, and is constant along the $x$ axis. The ion temperature is $T_{i}=80 \mathrm{eV}$. The electron temperature is $T_{e}=100 \mathrm{eV}$ for $\mathrm{PF} 3 \mathrm{D}$. The pump and seed wavelength is $1057 \mathrm{~nm}$, and their durations are, respectively, 1.7 and 0.55 ps (FWHM). Their focal spot sizes (FWHM) are $\sigma_{x} \times \sigma_{y} \simeq 85 \times 135 \mu \mathrm{m}^{2}$ and $\sigma_{x} \times \sigma_{y} \simeq 100 \times 36 \mu \mathrm{m}^{2}$. The pump peak intensity is $4 \times 10^{16}$, corresponding to an energy of $9 \mathrm{~J}$. The seed peak intensity is $10^{15} \mathrm{~W} / \mathrm{cm}^{2}$, corresponding to $E_{1}=40 \mathrm{~mJ}$ in Fig. 3 .

Figure 8(a) shows, at five different interaction stages, the spatial profiles of the pump and seed electric fields in the interaction plane ( $y-z$; cf. Fig. 1$)$. At $t=-0.7 \mathrm{ps}$, the seed pulse is in the plasma, it has not yet encountered the pump pulse, and its initial energy $(40 \mathrm{~mJ})$ and intensity are preserved (no significant losses). At $t=0$, the peak of the seed is in the middle of the plasma $(z=0)$, where the pump peak arrives $1 \mathrm{ps}$ later $\left(t_{0}-t_{1}=+1 \mathrm{ps}\right)$. The pump front, however, already starts to transfer its energy, and the peak intensity of the seed has increased by a factor of 4 $\left(I_{1} / I_{0 \max } \sim 0.1\right)$. At $0.4<z<0.8 \mathrm{~mm}$ (pump plasma entrance), one can see scattered light, which is spontaneous backward Brillouin of the pump, that starts to deplete its trailing edge. At $t=0.7 \mathrm{ps}$, the seed peak intensity is 120 times larger, reaching 3 times the incident pump one $\left(I_{1} / I_{0 \max } \sim 3\right)$. The pump profile shows a strong depletion with two patterns: one at its front dug by the seed (stimulated Brillouin) and an inhomogeneous one induced by spontaneous backward Brillouin at its back. Because of the $165^{\circ}$ interaction geometry, the seed front tends to bend and expand toward the pump front. The pump-seed overlap ends at $t=1.4 \mathrm{ps}$. When the seed leaves the plasma $(t=2.1 \mathrm{ps})$, its energy has reached $2.16 \mathrm{~J}$, close to the measured one (Fig. 3). After the interaction, the pump has undergone a strong depletion $(70 \%)$, close to what we measure (Fig. 3). Let us note that most of the energy transfer occurs within approximately $400 \mu \mathrm{m}$.

Figure 8(b) shows a 3D view of the amplified seed, revealing the $3 \mathrm{D}$ asymmetry. It corresponds to the same simulation as the 2D slices presented in Fig. 8(a), at time $t=1.7 \mathrm{ps}$ (exit of the plasma). The internal spatiotemporal structure of the pulse is visualized through the $y-z$ and $x-z$ plane cuts.

It is important to point out that, although the level of spontaneous scattering is significant, it is not enough by itself to justify the low transmission of the pump alone in our experiment, where other factors (discussed in Sec. III A) need to be taken into account. Thus, decreasing spontaneous scattering and increasing the quality of the created plasma are both important points to consider in order to increase the pump transmission and the energy available. The role of spontaneous instabilities and how to reduce them is discussed in the following.

\section{B. Influence of spontaneous pump backscattering}

Spontaneous Brillouin and Raman scattering have a significant influence on the results. Because of its faster 
(a)
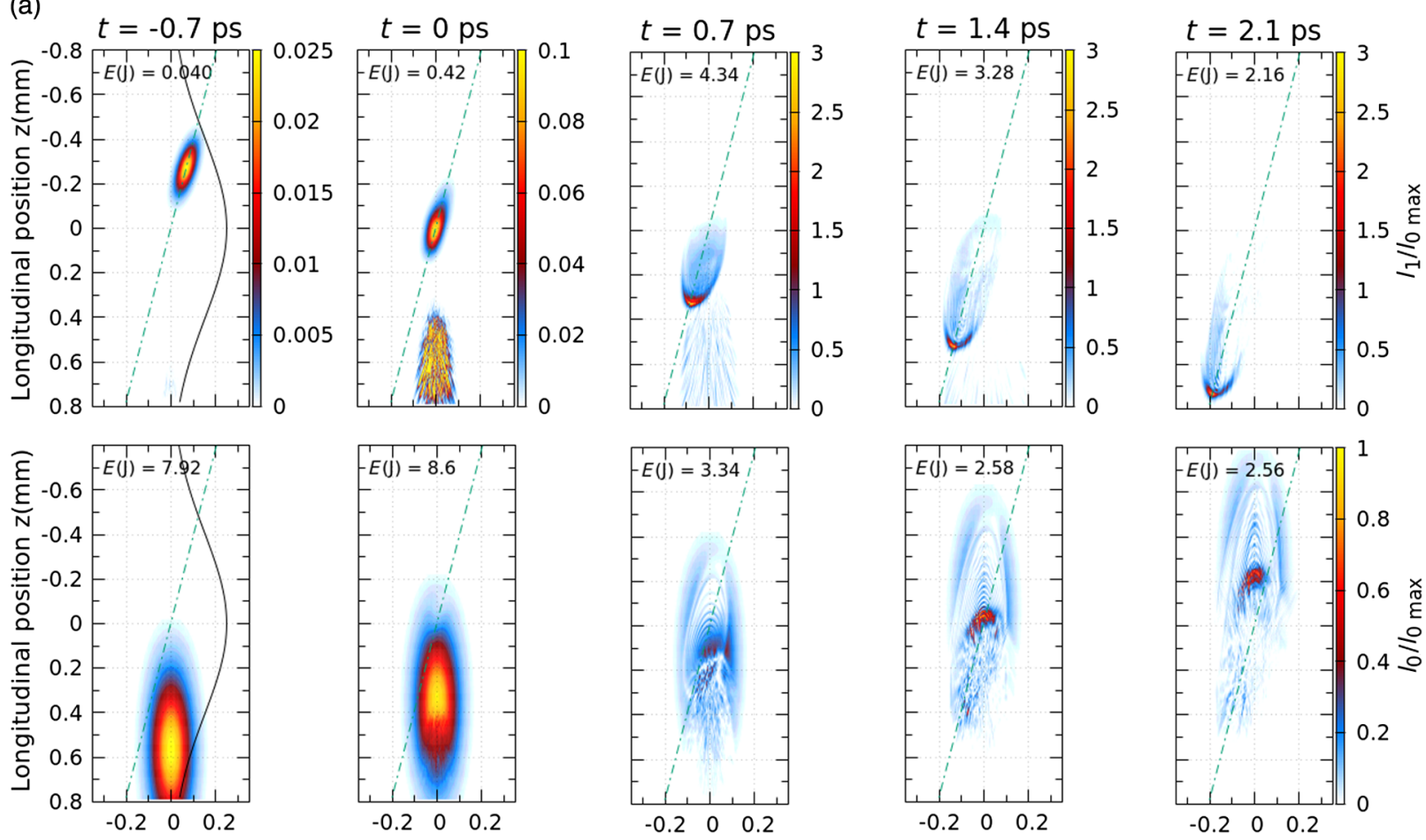

Transverse position $y(\mathrm{~mm})$

(b)

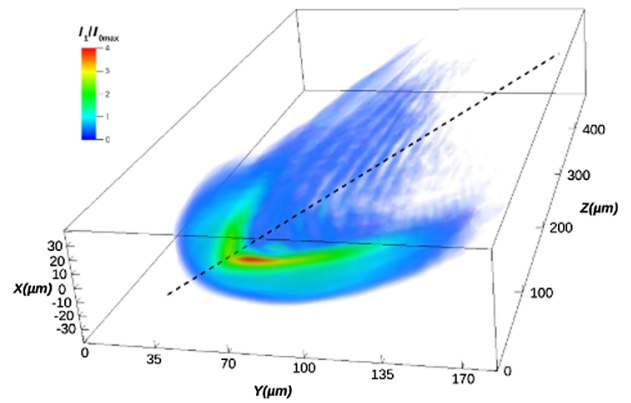

(c)

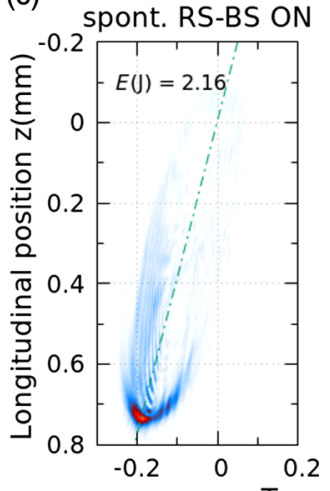

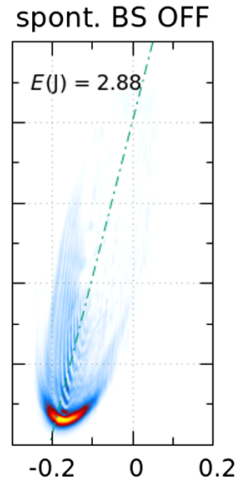

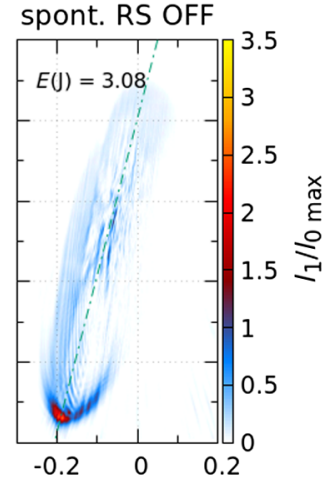

FIG. 8. Three-dimensional pump-seed interaction, $\mathrm{PF} 3 \mathrm{D}$ simulations. (a) Intensity profiles in the interaction plane $(y-z)$ of the seed [(a) upper images] and pump [(a) bottom images] pulses at different times (increasing from left to right). The green dashed line indicates the seed propagation axis ( $165^{\circ}$ from the pump). The black line in the left images indicates the plasma density profile along the $z$ axis. The pump arrives at the plasma center $1 \mathrm{ps}$ after the seed $\left(t_{0}-t_{1}=+1 \mathrm{ps}\right)$. Intensities are normalized to the incident pump maximum intensity $\left(I_{0 \max }=4 \times 10^{16} \mathrm{~W} / \mathrm{cm}^{2}\right)$. The laser-plasma parameters are the experimental ones; see the experimental setup section. (b) Three-dimensional intensity profile of the amplified seed that reveals the 3D asymmetry [the same laser-plasma parameters as in (a), at time $t=1.7 \mathrm{ps}$ (exit of the plasma)]. The internal spatiotemporal structure of the pulse is visualized through the $y-z$ and $x-z$ plane cuts. (c) Intensity profile in the $y-z$ plane of the seed pulse at the plasma exit $(t=2.1 \mathrm{ps})$, with [(c) left] or without spontaneous Brillouin [(c) center] and Raman [(c) right] instabilities. The central figure [(c) center] is without ion-acoustic fluctuations, SBS always on. The other parameters are the same as in (a).

growth rate, Raman is the most important. Because of an initial temperature $T_{e} \approx 100 \mathrm{eV}, k_{0} \lambda_{\mathrm{De}}=0.063$ and, with $k_{\mathrm{Lw}} \sim 1.6 k_{0}, k_{\mathrm{Lw}} \lambda_{\mathrm{De}}=0.1$, so Landau damping is unimportant at the peak density of $0.05 n_{c}\left(k_{0}\right.$ and $k_{\mathrm{Lw}}$ are the wave numbers of the laser and the Langmuir wave, respectively, and $\lambda_{\mathrm{De}}$ is the Debye length). Even at a lower density of $0.01 n_{c}, k_{\mathrm{Lw}} \lambda_{\mathrm{De}}=0.22$. $\mathrm{PF} 3 \mathrm{D}$ simulations show thata the plasma is heated to $T_{e}$ as high as $250 \mathrm{eV}$ when Raman is included. Nonetheless, Raman reaches saturation too fast for heating to affect the results. To study the 
influence of spontaneous Raman and Brillouin on the amplification process, we run 3D simulations with the SRS or the ion density noise turned off (stimulated Brillouin always on). This comparison is interesting even if a realistic treatment of the plasma fluctuations in envelope codes is not trivial, while in PIC codes the noise level is exaggerated and spontaneous backscattering overestimated on the short timescales used here. Figure 8(c) shows the spatiotemporal profile (intensity normalized to the pump maximum one) of the seed at the plasma exit obtained with $\mathrm{PF} 3 \mathrm{D}$ for three cases: with spontaneous Raman and Brillouin [Fig. 8(c), left, the same as Fig. 8(a)], without Brillouin [Fig. 8(c), center], and without Raman [Fig. 8(c), right]. The differences are explained as follows: At the plasma entrance, the ion density grating generated by spontaneous Brillouin scatters the pump light in its backward direction. At the plasma center, the angle between the two beams leads to a density grating that scatters the pump in the seed direction. Without spontaneous Raman, the ion grating is stronger and more energy is left at the back of the pump to be scattered in the seed direction, which forms the long tail observed in Fig. 8(c), right. Without spontaneous Brillouin, the ion grating has a more homogeneous spatial profile, which leads to a more homogeneous intensity profile of the amplified seed. Also, one can observe that the amplification process in crossed geometry tends to bend and extend the intensity distribution toward the pump axis $(y=0)$, as observed experimentally (Fig. 2). This tendency is induced by the combination of several factors. We have shown previously $[10,12]$ that the evolution of the phase that governs the three-wave interaction makes sc-SBS amplification more efficient if the seed propagates in a negative density gradient (from high to low density), so in our case in the $z>0$ side of the Gaussian density profile. We also observe (Fig. 7) that, by reducing the spontaneous pump scattering and thus preserving the pump energy, the maximum energy transfer occurs for pump-seed delay $t_{0}-t_{1}>0[+1 \mathrm{ps}$ in Fig. 8(a)], that is, for a seed that has already passed the plasma density peak before it overlaps the pump peak (again, the $z>0$ region). Finally, an important result of this paper (see Fig. 3) is that a significant energy exchange can still occur at a low seed incident intensity, so even the wings of the seed can be amplified. Combining these effects with the not-counterpropagating geometry $\left(165^{\circ}\right)$ leads to an asymmetric amplification process in favor of the $z>0$ region, with an energy transfer that tends to be larger toward $y=0$, where the pump intensity is maximum.

\section{Influence of the incident seed intensity}

We have performed a large number of simulations in order to study the dependence of the energy transfer on the seed incident intensity. The $\mathrm{PF} 3 \mathrm{D}$ envelope simulations have been completed by PIC simulations with the code
SMILEI [30] in a one-dimensional geometry. In the latter, even if the $165^{\circ}$ geometry of the interaction is not simulated, ion and electron nonlinearities (such as harmonics, trapping, or wave breaking) are included as opposed to the envelope code. Moreover, spontaneous Brillouin and Raman scattering are included in a selfconsistent way. The $\mathrm{PF} 3 \mathrm{D}$ and SMILEI simulations are run with the same laser-plasma input parameters, the experiment ones (see the experimental setup section). In the SMILEI simulations, the experimental pump frequency chirp $\left(\alpha=-7.7 \times 10^{-7}\right)$ is included, even if the results of the simulations indicate that it does not significantly modify the energy transfer in the present experiment. To take into account the heating by inverse bremsstrahlung absorption of the pump simulated in $\mathrm{PF} 3 \mathrm{D}, T_{e}$ is set at $300 \mathrm{eV}$ in the $1 \mathrm{D}$ PIC simulations with the pump and $100 \mathrm{eV}$ without. The PIC simulations are run with 300 particles per cell, giving at $T_{e}=300(100) \mathrm{eV}$ the spatial resolution better than 2.5(5) Debye length. The time step is $d t=0.175 \mathrm{fs}(0.95 d x / c)$. The initial positions of the pump and seed Gaussian pulses are adjusted depending on their relative delay $t_{0}-t_{1}$, the intensity of the rising edge at the entrance of the simulation box never exceeding $1 / e^{2}$ of their respective peak intensity. The boundary conditions are reflective for the particles and open ("silver-muller") for the electromagnetic waves.

Figure 9 shows the pump and seed energies at the plasma exit as a function of the seed incident intensity, for 1D PIC (left) and 3D-envelope ( $\mathrm{PF} 3 \mathrm{D}$, right) simulations, at $t_{0}-t_{1}=+1 \mathrm{ps}$. In both cases, one can observe the same behavior as the experimental results presented in Fig. 3: an increase of the transferred energy from the pump to the seed when the incident intensity is increased, up to an optimum. When the incident intensity is larger than the pump one, the initial conditions for optimum amplification are not met and the efficiency drops. In particular, the seed duration is too long for this configuration [10].

The dependency of the energy transfer on the incident seed intensity can be explained as follows [12]. During the coupling between the pump beam and the seed beam, the system successively explores different stages. In the initial stage, the phase of the seed adapts to the pump, but the energy transfer between the pump and seed is negligible. In the second stage, both the seed and the ion wave grow exponentially, and the energy exchange starts to be important when $I_{1} \sim I_{0}$. Pump depletion then becomes significant, and in this so-called self-similar regime, most of the remaining pump energy is transferred to the seed. The relevant parameter is thus the time and location at which pump depletion sets in. Indeed, from this time, the seed stores all the remaining part of the pump pulse: The earlier the pump depletion, the larger the energy gain. The larger its incident intensity, the faster the seed enters the exponential stage and grows to an intensity comparable to the pump, thus maximizing the energy gain. However, when $I_{1}$ is initially equal to $I_{0}$, the energy transfer from the pump to the seed takes place only in a 

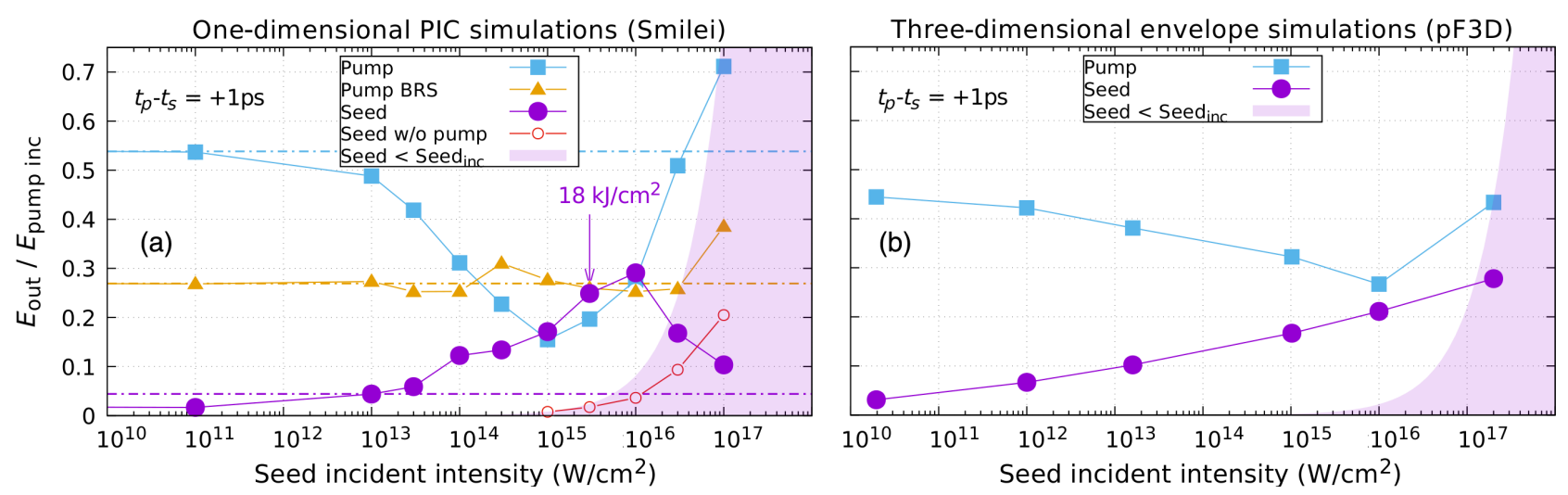

FIG. 9. Simulations, output energies versus seed incident intensity. (a) 1D PIC (SMILEI) and (b): 3D-envelope (PF3D) simulations, with the same laser parameters as in Fig. 8(a). The energies at the plasma exit of the pump and seed, as well as the pump backward Raman scattered light, are normalized to the incident pump one. The pump-seed delay is $t_{0}-t_{1}=+1 \mathrm{ps}$. The filled areas correspond to a seed output energy lower than the incident one (energy transfer from the seed to the pump). The dashed lines in (a) show the energies without a seed of spontaneous Brillouin, Raman, and transmitted pump (from bottom to top, respectively).

very small zone (wings of the seed), and an efficient gain requires a longer plasma and a longer pump duration. Notice that amplification of seeds more intense than the pump can be obtained from the beginning of the crossing in a different regime [24,25].

In $3 \mathrm{D}$, the interaction of the wings of the pump and seed beams occurs at a lower intensity than on axis, so that, on average, the maximum seed intensity at which the energy transfer is reversed (seed to pump) is larger than in 1D. From the 1D PIC simulations, one can see that without the seed, or at a low seed intensity, the spontaneous backward Raman and Brillouin scattering of the pump represent, respectively, $30 \%$ and $5 \%$ of the incident pump energy. However, when the incident seed intensity is increased, spontaneous Raman remains unchanged, while the energy transfer due to SBS becomes significant, up to $30 \%$ of the initial pump energy. Complementary simulations are performed increasing the ion mass by 100 . They confirm that the ions are the vector of the energy transfer from the pump to the seed: At an incident seed intensity of $I_{1}^{\text {inc }}=10^{15} \mathrm{~W} / \mathrm{cm}^{2}$ and $t_{0}-t_{1}=0$, the output seed has an energy 5.5 times lower and is twice longer. Accordingly, the transmitted pump energy is 1.8 times larger, while the Raman signal is almost unchanged.

In conclusion, both $1 \mathrm{D}$ and $3 \mathrm{D}$ approaches show the same behavior of the amplification process with the incident seed intensity and are in good agreement with the experimental results of Fig. 3. As an example, the energy per unit area obtained with the 1D PIC code $\left(18 \mathrm{~kJ} / \mathrm{cm}^{2}\right.$ for $\left.I_{1}=3 \times 10^{15} \mathrm{~W} / \mathrm{cm}^{2}\right)$ is in excellent agreement with the measured one. In addition, the relatively slow increase of the energy transfer with the incident seed intensity explains the increase of the spatial width of the amplified seed: The relative gain $\Delta E / E_{1}^{\text {inc }}$ of the lowintensity wings is larger than that of the high-intensity central part of the beam.

\section{Backward Raman scattering of the amplified seed}

In order to compare with the experimental results of Fig. 6, the Raman energy propagating backward from the seed (forward from the pump) is presented in Fig. 10, without or with the pump pulse, for the same set of simulations reported in Fig. 9(left). Almost no signal is generated when the pump is alone or the seed very weak. Without the pump (black curve), Raman becomes nonnegligible for incident seed intensities near or above $10^{16} \mathrm{~W} / \mathrm{cm}^{2}$. When the pump is on and the incident seed intensity is between $10^{14}$ and $10^{16} \mathrm{~W} / \mathrm{cm}^{2}$, the Raman signal increases, in correlation with the seed amplification observed in Fig. 9. At $I_{1}^{\text {inc }}=10^{15} \mathrm{~W} / \mathrm{cm}^{2}$, the signal is 1500 times larger, equivalent to the signal observed with the seed alone at an intensity 60 times larger (black curve). On the contrary, for $I_{1}^{\text {inc }}>10^{16} \mathrm{~W} / \mathrm{cm}^{2}$, the seed will transfer energy to the pump, thus decreasing the spontaneous seed backscattering.

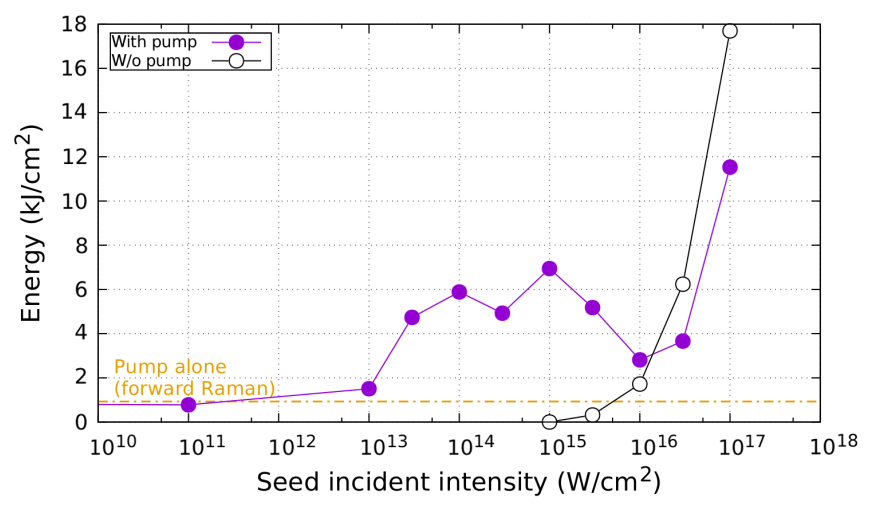

FIG. 10. 1D PIC simulations, backward Raman energy from the seed. The dashed orange line is the level of Raman without a seed, generated by forward Raman of the pump. The pump-seed delay is $t_{0}-t_{1}=+1 \mathrm{ps}$. 


\section{CONCLUSIONS AND PERSPECTIVES}

In this paper, laser-plasma amplification of subpicosecond pulses above the Joule level is demonstrated, a major milestone for this scheme to become a solution for the next generation of ultrahigh-intensity lasers. We demonstrate the importance of using a seed pulse of initial intensity high enough to enter the self-similar regime as promptly as possible and get a highly efficient (20\%) energy transfer in a subpicosecond scale. In the regime of efficient amplification, evidence of energy losses of the seed by spontaneous backward Raman was found. Simulations with a three-dimensional envelope code, supplemented with a one-dimensional particle-in-cell code, were compared with the experimental results. They show very good agreement with the amount of transferred energy and with the global behavior observed in the experiment. Spontaneous Raman, as well as spontaneous and seeded Brillouin, scattering reaches nonlinear levels. The pump transverse intensity profile, together with the crossed beam geometry, cause the seed intensity distribution to bend and extend toward the pump axis. This tendency could be avoided (limited) by using a pump beam with a larger focal spot or with a tilted intensity front [31] adjusted to the crossing angle. The output seed energy, intensity, and quality were limited by the homogeneity and extent of the spatiotemporal interaction volume, itself limited by the energy and quality of the laser beams. Without reducing the sc-SBS gain, the beam losses from spontaneous Raman scattering and collisional damping might be limited with the use of a plasma with a density ramp and a much higher temperature. An initially shorter seed pulse will also reduce these losses and would be better adjusted to the self-similar regime, increasing the pulse compression [10,23,32]. Much larger energy transfers might be reached using a longer pump and plasma, larger focal spots of homogeneous and hyper-Gaussian profiles. Exploiting more energetic lasers will allow these improvements and open the way to the numerically predicted [24,25] next step: an output seed intensity much larger than the pump one.

\section{ACKNOWLEDGMENTS}

We gratefully acknowledge the ELFIE technical staff for the invaluable help during the experiments, Mickael Grech, Frédéric Perez, and Tommaso Vinci for their help on the PIC code SMILEI, and Pascal Loiseau for fruitful discussions and 3D fluid simulations of plasma creation and heating by the ionization beam. This work has been done within the LABEX Plas@par project and received financial state aid managed by the Agence Nationale de la Recherche, as part of the program "Investissements d'avenir" under Reference No. ANR-11-IDEX-0004-02. S. W. was supported by the projects HIFI (High Field Initiative, CZ.02.1.01/0.0/0.0/15_003/0000449) and ADONIS (ADvanced research using high intensity laser produced phOtons aNd partIcleS, CZ.02.1.01/0.0/0.0/ 16_019/0000789), both from European Regional Development Fund. M. B. was supported by a grant from GSI (Gesellschaft für Schwerionenforschung $\mathrm{mbH}$ ). The research leading to these results has received funding from LASERLAB-EUROPE, Grant Agreement No. 284464 , EC's Seventh Framework Program. L. L. was supported by CRISP, Contract No. 283765, EC's Seventh Framework Program. The work of R. B. was performed under the auspices of the U.S. Department of Energy by Lawrence Livermore National Laboratory under Contract No. DEAC52-07NA27344.

[1] D. Strickland and G. Mourou, Compression of Amplified Chirped Optical Pulses, Opt. Commun. 56, 219 (1985).

[2] T. Tajima and G. Mourou, Zettawatt-Exawatt Lasers and Their Applications in Ultrastrong-Field Physics, Phys. Rev. Accel. Beams 5, 031301 (2002).

[3] V. M. Malkin, G. Shvets, and N. J. Fisch, Fast Compression of Laser Beams to Highly Overcritical Powers, Phys. Rev. Lett. 82, 4448 (1999).

[4] Y. Ping, I. Geltner, N. J. Fisch, G. Shvets, and S. Suckewer, Demonstration of Ultrashort Laser Pulse Amplification in Plasmas by Counterpropagating Pumping Beam, Phys. Rev. E 62, R4532 (2000).

[5] D. S. Clark, Operating Regime for a Backward Raman Laser Amplifier in Preformed Plasma, Phys. Plasmas 10, 3363 (2003).

[6] R. D. Milroy, C. E. Capjack, and C. R. James, Plasma Laser Amplifier Using Induced Raman or Brillouin Processes, Phys. Fluids 22, 1922 (1979).

[7] D. W. Forslund, J. M. Kindel, and E. L. Lindman, Theory of Stimulated Scattering Processes in Laser-Irradiated Plasmas, Phys. Fluids 18, 1002 (1975).

[8] A. Andreev, C. Riconda, V. T. Tikhonchuk, and S. Weber, Short Light Pulse Amplification and Compression by Stimulated Brillouin Scattering in Plasmas in the Strong Coupling Regime, Phys. Plasmas 13, 053110 (2006).

[9] L. Lancia et al., Experimental Evidence of Short Light Pulse Amplification Using Strong-Coupling Stimulated Brillouin Scattering in the Pump Depletion Regime, Phys. Rev. Lett. 104, 025001 (2010).

[10] M. Chiaramello, C. Riconda, F. Amiranoff, J. Fuchs, M. Grech, L. Lancia, J.-R. Marquès, T. Vinci, and S. Weber, Optimization of Interaction Conditions for Efficient Short Laser Pulse Amplification by Stimulated Brillouin Scattering in the Strongly Coupled Regime, Phys. Plasmas 23, 072103 (2016).

[11] M. R. Edwards, Q. Jia, J. M. Mikhailova, and N. J. Fisch, Short-Pulse Amplification by Strongly Coupled Stimulated Brillouin Scattering, Phys. Plasmas 23, 083122 (2016).

[12] F. Amiranoff, C. Riconda, M. Chiaramello, L. Lancia, J. R. Marquès, and S. Weber, The Role of the Global Phase in the Spatio-Temporal Evolution of Strong-Coupling Brillouin Scattering, Phys. Plasmas 25, 013114 (2018).

[13] M. Chiaramello, F. Amiranoff, C. Riconda, and S. Weber, Role of Frequency Chirp and Energy Flow Directionality in 
the Strong Coupling Regime of Brillouin-Based Plasma Amplification, Phys. Rev. Lett. 117, 235003 (2016).

[14] G. Lehmann and K. H. Spatschek, Control of Brillouin Short-Pulse Seed Amplification by Chirping the Pump Pulse, Phys. Plasmas 22, 043105 (2015).

[15] F. Schluck, G. Lehmann, and K. H. Spatschek, Amplification of a Seed Pumped by a Chirped Laser in the Strong Coupling Brillouin Regime, Phys. Plasmas 23, 083105 (2016).

[16] L. Lancia et al., Signatures of the Self-Similar Regime of Strongly Coupled Stimulated Brillouin Scattering for Efficient Short Laser Pulse Amplification, Phys. Rev. Lett. 116, 075001 (2016).

[17] D. Turnbull, S. Bucht, A. Davies, D. Haberberger, T. Kessler, J. L. Shaw, and D. H. Froula, Raman Amplification with a Flying Focus, Phys. Rev. Lett. 120, 024801 (2018).

[18] Q. Chen, Z. Wu, A. Morozov, and S. Suckewer, Stimulated Raman Near-Backscattering with a Pulse-Front-Tilted Pump, Phys. Plasmas 25, 093110 (2018).

[19] J. D. Sadler et al., Advantages to a Diverging Raman Amplifier, Commun. Phys. 1, 19 (2018).

[20] J. D. Sadler et al., Optimization of Plasma Amplifiers, Phys. Rev. E 95, 053211 (2017).

[21] G. Vieux et al., An Ultra-High Gain and Efficient Amplifier Based on Raman Amplification in Plasma, Sci. Rep. 7, 2399 (2017).

[22] P. Loiseau (private communication).

[23] R. M. G. M. Trines et al., Essential Criteria for Efficient Pulse Amplification via Raman and Brillouin Scattering, arXiv:1611.04485.

[24] C. Riconda , S. Weber, L. Lancia, J.-R. Marquès, G. Mourou, and J. Fuchs, Plasma-Based Creation of Short
Light Pulses: Analysis and Simulation of Amplification and Focusing, Plasma Phys. Controlled Fusion 57, 014002 (2015).

[25] S. Weber, C. Riconda, L. Lancia, J. R. Marques, G. A. Mourou, and J. Fuchs, Amplification of Ultrashort Laser Pulses by Brillouin Backscattering in Plasmas, Phys. Rev. Lett. 111, 055004 (2013).

[26] E. P. Alves et al., A Robust Plasma-Based Laser Amplifier via Stimulated Brillouin Scattering, arXiv:1311.2034v3.

[27] Z. M. Zhang, B. Zhang, W. Hong, Z. G. Deng, J. Teng, S. K. He, W. M. Zhou, and Y. Q. Gu, Generation of High-Power Few-Cycle Lasers via Brillouin-Based Plasma Amplification, Phys. Plasmas 24, 113104 (2017).

[28] R. L. Berger, B. F. Lasinski, T. B. Kaiser, E. A. Williams, A. B. Langdon, and B. I. Cohen, Theory and ThreeDimensional Simulation of Light Filamentation in LaserProduced Plasma, Phys. Fluids B 5, 2243 (1993).

[29] R. L. Berger, C. H. Still, E. A. Williams, and A. B. Langdon, On the Dominant and Subdominant Behavior of Stimulated Raman and Brillouin Scattering Driven by Nonuniform Laser Beams, Phys. Plasmas 5, 4337 (1998).

[30] J. Derouillat et al., Smilei: A Collaborative, Open-Source, Multi-Purpose Particle-in-Cell Code for Plasma Simulation, Comput. Phys. Commun. 222, 351 (2018).

[31] S. Akturk, M. Kimmel, P. O'Shea, and R. Trebino, Measuring Pulse-Front Tilt in Ultrashort Pulses Using GRENOUILLE, Opt. Express 11, 491 (2003).

[32] R. M. G. M. Trines et al., Boosting the Performance of Brillouin Amplification at Sub-Quarter-Critical Densities via Reduction of Parasitic Raman Scattering, arXiv: $1406.5424 \mathrm{v} 1$. 